\title{
A Mixed-Methods Evaluation of Nonverbal Communication in Collaborative Video Games
}

\author{
Aaron May \\ School of Interactive Arts \\ and Technology \\ Simon Fraser University \\ Surrey BC, Canada \\ amay@sfu.ca
}

\author{
Amber Choo \\ School of Interactive Arts \\ and Technology \\ Simon Fraser University \\ Surrey BC, Canada \\ achoo@sfu.ca
}

\author{
Carman Neustaedter \\ School of Interactive Arts \\ and Technology \\ Simon Fraser University \\ Surrey BC, Canada \\ carman_neustaedter@sfu.ca
}

\author{
Alissa Antle \\ School of Interactive Arts \\ and Technology \\ Simon Fraser University \\ Surrey BC, Canada \\ aante@sfu.ca
}

\begin{abstract}
In order to explore the benefits of communication in complex settings such as work and home environments, it is important to have a firm understanding of how communication operates in a relatively controllable system, such as video games. This paper aims to evaluate the impact of nonverbal communication on success during collaborative gameplay using both qualitative observations of and quantitative gameplay data from players of the custom-designed application TeamWyrm. In so doing, it encourages a discussion of nonverbal communication's role in gameplay, the forms of nonverbal communication displayed during gameplay, and how the availability of nonverbal communication affects player performance.
\end{abstract}

Keywords- Communication; video games; collaboration; nonverbal

\section{INTRODUCTION (Heading 1)}

Communication is a complex concept being studied through a number of lenses, including professional, academic, and domestic domains. Communication processes benefit the growth of an individual's knowledge, interpersonal skills, multiculturalism, professional identity, and ability to integrate with a team. One domain that relies heavily on communication for user advancement is that of collaborative video games. Communication is especially important to players of teamfocused games from the Massively Multiplayer Online Role Playing Games (MMORPG), First-Person Shooters (FPS), and Real-Time Strategy (RTS) genres [13]. Improved understanding of communication in collaborative video games can help gain insight into other domains and can drive improvements within video game play.

Nonverbal communication takes a variety of forms. Traditional psychology classification schemes break nonverbal communication into facial expression, gestures, paralinguistics, body language and posture, proxemics, eye gaze, haptics, and appearance [5]. This study focuses on the gestural aspect of nonverbal communication, and will use the two synonymously. This simplification was made to focus on one form of nonverbal communication and ease data collection.
During cooperative and collaborative play, opportunities for communication frequently arise when individual players are presented with only a portion of the data involved in play. This information imbalance heavily influences the gameplay of many card and tabletop games. For example, Go Fish works because players are able to declare a card type to others. Pictionary wouldn't be much of a game if the player couldn't draw for their teammates. A Dungeons \& Dragons player relies on the spatial relationships between his cleric and the other party members to perform miraculous feats of healing. Multiplayer video games focus heavily on cooperative and collaborative gaming. The only way to "Catch "Em All" in the original Pokémon Red and Pokémon Blue was to trade with friends who had the opposite "color" game as you. Players tackling the Halo campaign on a split television screen could ascertain their partner's vital statistics with a quick glance. Even coordinating a score-multiplying Overdrive on Rock Band can be as simple as a quickly stated, "Ready? Go." to the drummer seated on the couch.

However, differences exist between video games with online and offline multiplayer. When playing in the same physical space as teammates, there are greater opportunities to communicate outside the game's designed systems. Playing a game like Team Fortress 2 online requires the use voice or text chat in conjunction with graphical player representations to ascertain the positioning of teammates. Playing the same game right next to a teammate provides greater affordances. Players can point, gesture, and perhaps even look at one-another's screens.

It is the knowledge of the nonverbal communication channel during collocated collaborative gameplay that this study intends to expand. Theoretically, players with additional communication channels should perform better in a collaborative game. This assumption draws on Tang, et al.'s conclusions showing the importance of timeliness and spatial context to collaboration in First Person Shooter games combined with research on nonverbal communication from other domains, including Pease and Pease and Knapp and Hall $[13,10,7]$. Taking that further, nonverbal communication offers gestural options that don't have strong analogues in 
other communication channels. For example, the voice command substitute for pointing downward requires more time to convey and features greater delay in processing. For these reasons, blocking nonverbal communication should have a negative impact on users' performance in the collaborative game TeamWyrm.

\section{RELATED WORK}

Tanenbaum, et al. investigated three perspectives (Information Deficit, Procedural Rhetoric, and Emergent Dialogue) to persuasion and behavior change used in serious games [12]. Understanding the pros and cons of the three approaches to persuasion and behavior change in games is valuable for understanding how communication within and around gameplay can impact a player's behavior.

Yun et al. investigated the impact of geographic proximity on communication in Massively Multiplayer Online Role Playing Games. They found that proximity did have an impact on in-game communication, particularly partner relationships [17]. Because one of this study's conditions uses a literal and metaphorical gulf, communication should suffer as a result.

Pena and Hancock compared the socioemotional and taskoriented content of in-game text messages sent during sessions of online video games in order to make comparisons with existing research of more instrumental computer-mediated communications. Their study found that more socioemotional messages were sent than task-oriented ones. Also, the most experienced users sent far more positive socioemotional messages than less experienced ones [10]. It is important to note that their results indicate a large part of in-game communication is not task-oriented.

Tang, et al. explored how First Person Shooter players use a shared voice channel to coordinate actions. Their findings emphasize the difficulty in coordinating during collaborative gameplay and suggest that timeliness and spatial context are essential to proper collaboration [13]. It is expected that this study's participants will likewise rely on timing and spatial cues to convey accurate directions. How these cues will translate to nonverbal commands may be discovered.

Hogan and Stubbs claimed that nonverbal communication makes up $2 / 3$ of all interpersonal communication. However, communication can result when nonverbal communication contradicts verbal communication [6]. This turns out to figure prominently in this study's results.

Dunning summarized the state of nonverbal communication and upholds the categories of facial expression, gestures, paralinguistics, body language and posture, proxemics, eye gaze, haptics, and appearance [5]. This study will focus primarily on gestures.

Pease and Pease summarized the work of nonverbal communication pioneers such as Ray Birdwhistell [10]. Of greater importance to this study, they provide some categorization for gestures and their applicability across cultures. A future extension of my research may compare collaborative game performance within or across cultural groups.
Adding to that, Knapp and Hall claimed that nonverbal gestures can be categorized in terms of their relationship to speech. Gestures unassociated with speech have can be translated verbally, but gestures used while talking are usually used for emphasis and lack independent communicative power [7]. Most of the gestures displayed by participants in this study are dependent on speech.

\section{RESEARCH INSTRUMENT}

A collaborative game was required in order to assess the role of nonverbal communication in such a setting. TeamWyrm was developed in Processing as a multiplayer variation of the classic game Snake. Snake originated as the 1976 arcade game Blockade, but has gone by a number of names and variations, including home gaming consoles, graphing calculators, and cellular phones. In Snake, the player's goal is to maneuver the snake around a bordered space while eating the randomly spawned food. As more food is eaten, the snake increases in length and speed. Players fail when they contact either the border of the play area or the snake's "tail."

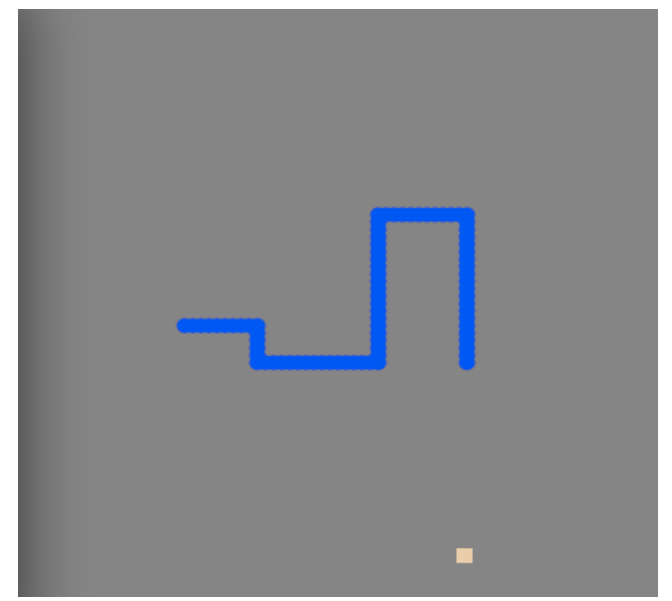

Fig. 1. Snake gameplay. Player controls the Snake (blue) to eat food (yellow) while avoiding the tail and boundary

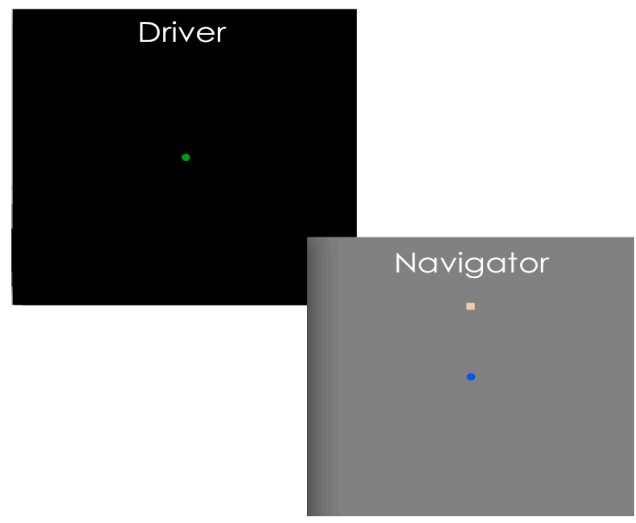

Fig. 2. In TeamWyrm, the food only appears on the Navigator's window. 
TeamWyrm modifies the classic Snake formula by incorporating an additional player. Each player has a separate application window and role, "driver" or "navigator." The driver has command of the snake and can see the play area and the snake. The navigator can see the play area, the snake, and the food; and is responsible for communicating the location of the food to the driver. Success in TeamWyrm requires effective communication between the driver and navigator. By including collaborative play, opportunities for verbal and nonverbal communication, co-locative play, and the reliance on communication for success, TeamWyrm's design maps to the key features of many collaborative games, making the results of this study generalizable to the broader domain.

\section{STUdy Design}

TeamWyrm was running on a laptop arranged back-to-back with a connected external display. The driver's window appeared on the laptop's screen and the navigator's window appeared on the external display. Players were seated facing each other to ensure a clear pathway for nonverbal communication. One option considered was to seat players side-by-side. However, with the face-to-face orientation, navigator gestures visually occurred "around" the driver's screen. This required less head and eye movement to encounter the nonverbal output and allowed for greater focus on gameplay. The two study conditions (and independent variable) were "Clear", with the nonverbal communication channel available; and "Obstructed", with the nonverbal communication channel unavailable. The blocking of nonverbal communication occurred by placing a large object between the laptop and external display, completely eliminating the sightline between driver and navigator.

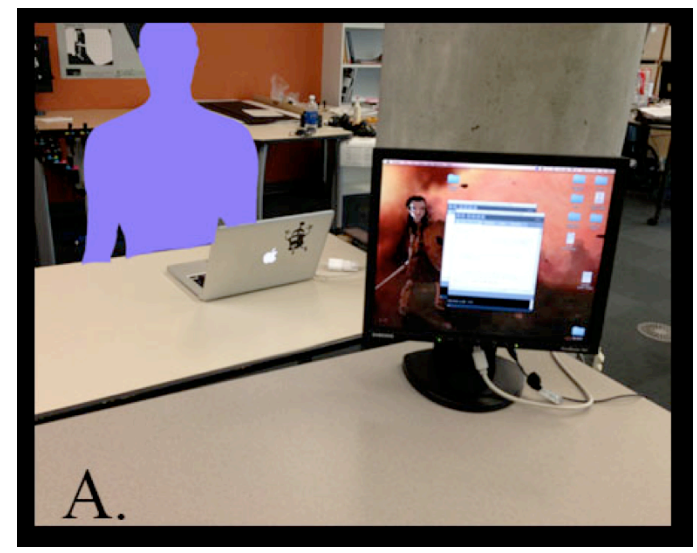

Fig. 3. View from the navigator's seat with nonverbal communication open (Clear condition).

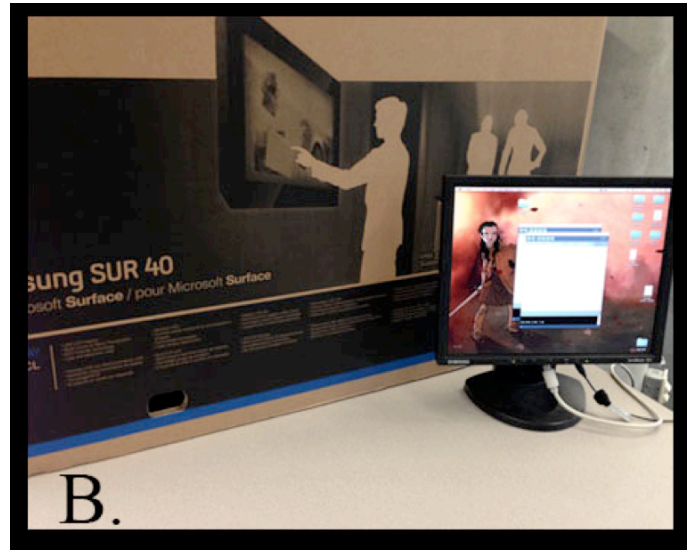

Fig. 4. View from the navigator's seat with nonverbal communication closed (Obstructed condition).

40 total individuals participated in the study (20 per condition). Study participants played TeamWyrm under one of the conditions in pairs for two sequential sessions and then completed a questionnaire. Participants were told that assessing communication was the aim of the study, but were not told anything about nonverbal communication, avoiding a Hawthorne effect.

Each play session was composed of a 4-minute practice portion during which performance was not recorded, followed by a 5 -minute measured portion. Participants were encouraged to use the practice session to become familiar with the controls of the game and refine communication strategies. During the measured portion, the researcher logged instances of food eaten, instances of failure, the reason for failure, navigation strategies, requested navigation strategies, and use of nonverbal communication. The score for a given participant (or, often, pair of participants) is the number of food eaten during the measured portion. After the first session, participants switched spots to practice and play in the other role (driver became navigator and vice versa). Audio was recorded during the play sessions for the researcher to reference later.

The post-play questionnaire collected participants' basic demographic data (sex, age), experience ("Do you play video games?" "Had you played Snake prior to this experiment?"), and degree of familiarity with their partner ("Had you worked with your partner prior to this experiment?" "Had you played games with your partner prior to this experiment?"). It also assessed the level of difficulty ("How challenging was it to succeed at the game?") and importance of communication ("How important was team communication to your success?") with five-point Likert scales. Finally, a space was provided for additional thoughts and comments.

\section{RESUlts}

Participant demographics were nicely split between the two conditions. They had an identical number of males and females (11 males, 9 females in each group), and that ratio was 
close to even ( $55 \%$ male, $45 \%$ female). Average age was close (26.5 years for Clear, 25.05 years for Obstructed).

User experience was also very comparable. Each condition had an equal number of self-identified video game players (14 of 20 for $70 \%$ ). $95 \%$ of the Clear participants had experience with Snake (19 of 20), compared to $90 \%$ of the Obstructed participants (18 of 20).

The final demographic concern, the level of familiarity between partners, was slightly less even but still very close. $70 \%$ of the pairs in the Clear condition had worked together before ( 7 of 10), compared to $50 \%$ for the Obstructed condition (5 of 10). $40 \%$ of the pairs in the Clear condition had previously played games together (4 of 10), compared to $30 \%$ of the pairs for the Obstructed condition (3 of 10).

In total, the demographic data reveals that each condition's participant pool was very similar. If anything, the Clear condition may have a small advantage on account of their slightly larger number of game players, Snake players, and experience within pairs. The Obstructed condition held a slight advantage in terms of youth.

Likert ratings for game difficulty and the importance of communication were similarly close. On average, participants in the Obstructed condition felt the game was slightly more challenging ( 3.25 out of 5 compared to 3 for the Open). The average importance given to communication was the same for participants in both conditions (4.9 out of 5).

When it came to TeamWyrm performance, the results were unexpected. The Obstructed condition posted the highest score (69, versus Clear's high of 55), a higher mean score (42, SD = 13.52 versus $40.1, \mathrm{SD}=9.36)$, and only a slightly lower lowest score (27 versus 28). (Fig. 5). A T-test reveals $\mathrm{p}>0.05$. Additionally, the Obstructed condition failed 13\% less frequently (39 to 45 ) than the Open condition. (Fig. 6)

Those results are for the group as a whole. However, not all pairs used nonverbal communication during gameplay. Only 13 of 20 Navigators used nonverbal events, and their distribution were pretty even between the conditions ( 6 for Clear, 7 for Obstructed). (Fig. 7). The resulting average scores from the nonverbal-assisted navigation were in favor of the Obstructed condition (21 versus 18.167 for the Clear condition). The nonverbal-assisted average scores were $9 \%$ lower than the within-condition average score for Clear (18.167, SD $=5.49$ compared to 20.05) and dead even for Obstructed (21 for both with nonverbal assisted SD $=8.75$ ). (Fig. 8).

Delving even deeper, thematically analyzing nonverbal events found that they fit into one of three categories: Pointing (navigator points in a direction to correspond with the needed movement), Timing (used to signal the moment when an action needs to occur), and Expression (used for exaggerated display of emotion). No condition/category set had more than 3 occurrences or less than 1. Pointing was most common in the Clear condition, and Timing was most common in the Obstructed condition (3 instances each). (Fig. 9). The only condition/category sets to outperform the condition average were Clear/Timing $(20.5, \mathrm{SD}=1.41$ versus 20.05$)$ and Obstructed/Pointing (24, SD = 10.61 versus 21). (Fig. 10).

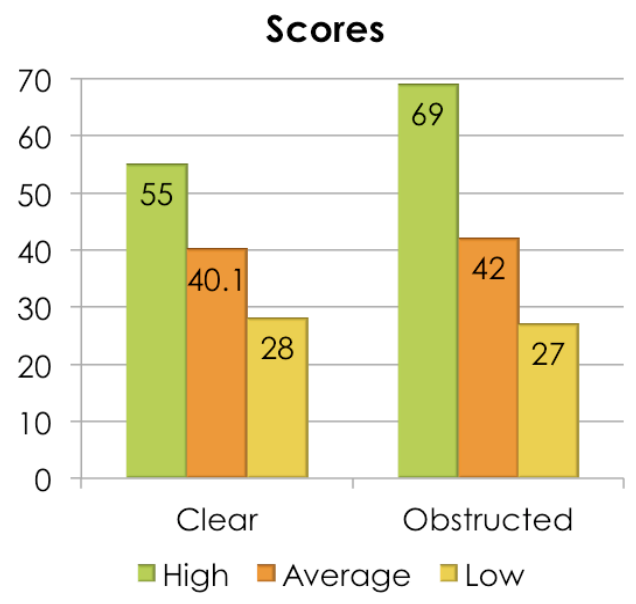

Fig. 5. High, Average, and Low scores per condition.

\section{Failures}

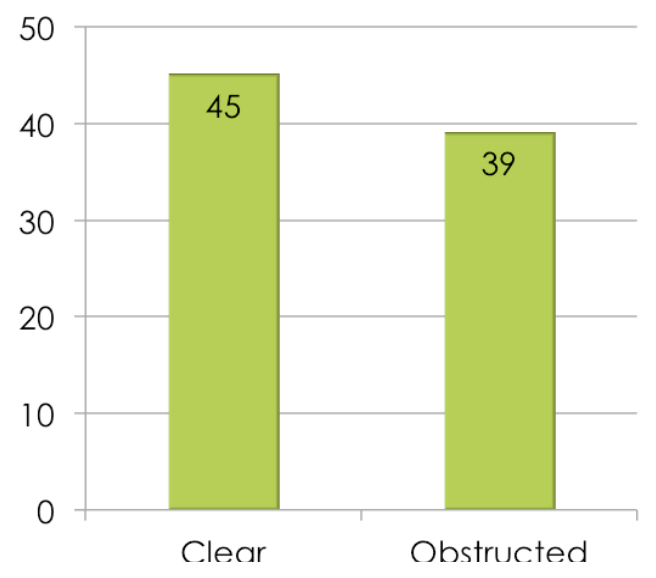

Fig. 6. Number of failures per condition.

\section{Nonverbal Events}

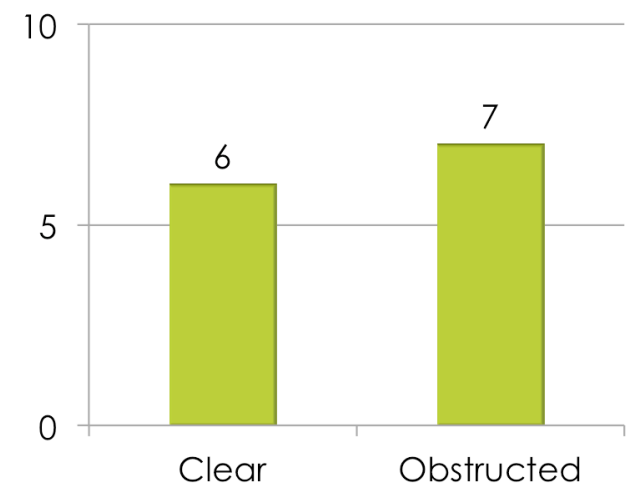

Fig. 7. Nonverbal events per condition. 


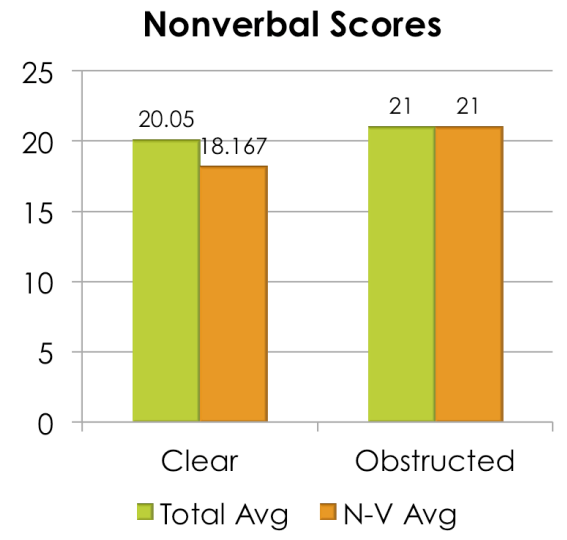

Fig. 8. Nonverbal average scores compared to total average scores per condition per condition.

\section{NV Category Events}

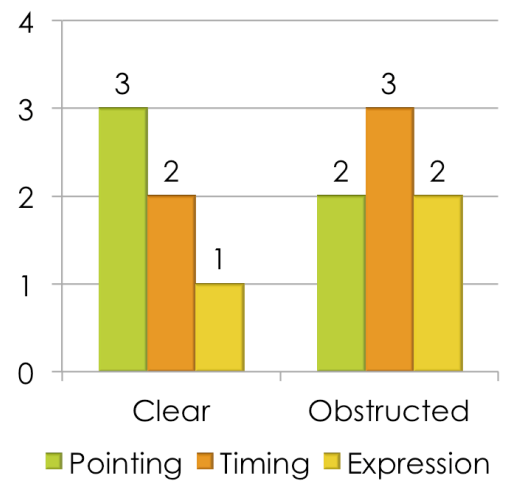

Fig. 9. Nonverbal events by category and condition.

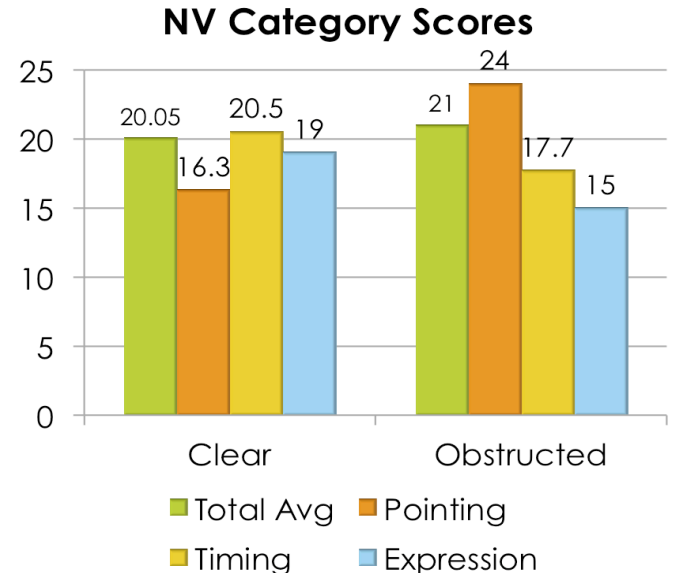

Fig. 10. In TeamWyrm, the food only appears on the Navigator's window.

\section{DISCUSSION}

We did not find a statistically significant difference in performance scores between players who were allowed to use the non-verbal communication channel. This suggests that players are able to perform equally well without non-verbal communication

If one were to think about designing systems, and game systems specifically, a large design addition would be creating features that explicitly provides players with ways to communicate non-verbally. These results show that this type of addition may actually not be that valuable because it didn't improve player scores. However, it also didn't make them any worse, at least there is no statistically significant difference to suggest non-verbal communication reduces performances. So, designing to include non-verbal communication could be more to appease certain players who simply "like" to communicate that way. This could be akin to the way some people like to "talk with their hands" or use non-verbal communication more explicitly than others. Thus, designing for it may be simply focusing on personal preferences rather than real performance needs

Slightly more nonverbal events occurred during the Obstructed condition, but these could not have impacted gameplay because the activity did not pass through from the navigator to the driver. Identical average scores between the instances with nonverbal activity and the total condition score backs up this assertion.

Where things get interesting is in comparing the distribution of the nonverbal category average scores. The sample sizes are small, so it is impossible to draw definitive conclusions. However, we see that within the Clear condition, the lowest average scores (16.3) were caused by the most frequent activity (Pointing). Why might Pointing be problematic? Because the orientation of the players makes pointing in a left-or-right direction counterproductive. If the food is on the right hand side of the navigator's screen, it also appears on the right hand side of the driver's screen relative to each player's perspective. However, when a navigator physically points to the right of their screen, the driver (facing them) sees that gesture occur to the left of their screen. This is the same reason that writing appears backwards in a mirror. In contrast, the category that produced the highest scores (20.5) in the Clear condition, Timing, has no orientation problems. Seeing the drop of a hand acts as a useful cue, and is even perceptible in peripheral vision.

What about the relatively high score (24) associated with Pointing actions in the Obstructed condition? It is possible that Pointing helps the navigator verbalize commands. With the nonverbal communication channel blocked, the gestures aid instruction without providing conflicting information to the driver. 


\section{LIMITATIONS}

The unexpected insignificance of nonverbal communication's impact on success in TeamWyrm yields three possible explanations.

First, it is possible that nonverbal communication just doesn't matter, especially in this context. In the open feedback section of the questionnaire, several users commented on the intensity and anxiety they felt when playing the game. Qualitative observation backs this up- drivers were very focused on controlling the snake. Their focus on avoiding failure may have led them to completely ignore the nonverbal cues offered by the navigator. It is possible that a less-intense research instrument would provide data that verifies this explanation. Also, overlaying the visual data on the driver's screen such as in the Videoarms system by Tang, et al. [14] may put it in a "position" that is easier to absorb.

Second, it is possible that discernable nonverbal communication actually harms success. This would explain the Obstructed condition's highest score, higher average scores, and reduced rate of failure. It explains why there was a performance discrepancy in the Clear condition between drivers who received nonverbal commands and the others. That there was no discrepancy between the same groups in the Obstructed condition only adds to the evidence. And the details of the action categories lend further weight. Drivers who could see Pointing fared significantly worse than those who couldn't. They posted the lowest average scores in the Clear condition. It is possible that changing the orientation of the players or the visual data so that left-right Pointing commands appear properly for the driver would negate these effects. Arranging the navigator in front of the driver, with their back to the driver would be one way to accomplish this.

Finally, there is a possible explanation buried deep within the participant demographics. The questionnaire wasn't designed to reveal this information, but it was something I grew concerned of as the study progressed. A number of the participants were students drawn from a third-year undergraduate video game design class at Simon Fraser University. By chance, $100 \%$ of these participants ended up in the Obstructed condition. A series of assumptions cascades to a potential reason for the study results. Being enrolled in a game design class, these students are likely very interested in video games, and they play them quite a bit (both individually and with others). These individuals may have been uniquely equipped to succeed at TeamWyrm. Because they were distributed entirely into the Obstructed condition, this expertise may have led to inflated scores compared to the Clear participants. How great could this impact have been? It is impossible to tell. However, it is worth noting that members of this class set the two highest scores in the Obstructed condition (and, consequently, the entire study). A pair of individuals not from the class set the lowest score in the Obstructed condition.

The research instrument may have also played a part in the findings. A game where communication predominantly flows in one direction (from the navigator to the driver) and where one player's agency is, to a great extent, removed (the driver is largely dependent on the navigator's commands to find the food) may not be the best way to test the importance of nonverbal communication in a collaborative game. Balancing either or both of those inequalities may lead to significantly different results.

The next steps to this research are to re-run the study while ensuring more even distribution of the super-experienced users between the conditions and correcting for the problematic Pointing. An overlay on the driver's display showing the navigator's hand gestures in proper orientation is a good place to start.

\section{CONCLUSION}

Through a mixed methods research study, nonverbal communication was found to not have a positive impact on user performance in the collaborative video game TeamWyrm. In fact, the collected data indicates that nonverbal communication may negatively impact success. Further investigation of this phenomena is required to extrapolate knowledge solid enough to apply to other contexts. A possible first avenue for continuing my research is to revise the study layout so that Pointing commands are more beneficial to the driver.

\section{REFERENCES}

[1] Appley, Dee G., and Alvin E. Winder. An evolving definition of collaboration and some implications for the world of work. The Journal of Applied Behavioral Science 13.3 (1977)

[2] Beale, R.. What makes a good game?: using reviews to inform design. Proceedings of the 23rd British HCI Group Annual Conference on People and Computers: Celebrating People and Technology. British Computer Society, (2009).

[3] Creswell, J. W. Qualitative inquiry and research design: Choosing among five approaches. SAGE Publications, Incorporated, (2012).

[4] De Waal, Brent. Motivations for video game play: a study of social, cultural and physiological factors. Diss. Theses (School of Communication)/Simon Fraser University, (1995).

[5] Dunning, G. B. Research in nonverbal communication. Theory into Practice 10, no. 4. (1971).

[6] Hogan, K. and Stubbs, R. Can't Get Through: Eight Barriers to Communication. Pelican Publishing, (2003).

[7] Knapp, M. L., and Hall, J.A. Nonverbal communication in human interaction. Wadsworth Publishing Company, (2009).

[8] Sherry, J.L. Sex differences in video game play: A communicationbased explanation. Communication Research 31.5 (2004).

[9] Pease, B. and Pease, A. The definitive book of body language. Bantam, (2008).

[10] Pena, J. and Hancock, J.T. An Analysis of Socioemotional and Task Communication in Online Multiplayer Video Games. Communication Research, Vol. 33, No. 1 (2005).

[11] Ross, J. and Tomlinson, B. How Games Can Redirect Humanity's Cognitive Surplus for Social Good. ACM Computers in Entertainment, Vol. 8, No. 4, Article 25, (2010).

[12] Tanenbaum, J., Antle, A.N., and Robinson, J. Three Perspectives on Behavior Change for Serious Games. CHI '13, (2013).

[13] Tang, A., Massey, J., Wong, N., Reilly, D., and Edwards W.K. Verbal coordination in first person shooter games. Proceedings of the ACM 2012 conference on Computer Supported Cooperative Work. ACM, (2012).

[14] Tang, A., Neustaedter, C., and Greenberg, S. Videoarms: embodiments for mixed presence groupware. People and Computers XX-Engage. Springer London, (2007).

[15] Walther, J. B. Computer-mediated communication impersonal, interpersonal, and hyperpersonal interaction. Communication research 23.1 (1996). 